\title{
Some Aspects of Mathematics and Its Applications
}

\author{
Savita \\ Asstt. Professor in Mathematics, Hindu College, Sonepat
}

\begin{abstract}
The purpose of this paper is to assess the attitude, knowledge and practice of mathematics in education. At different times and in different cultures and countries, mathematics education has attempted to achieve a variety of different objectives. In this paper, some aspects of mathematics are discussed. Starting with the definition of mathematics given by some of the pioneers of the field, an introduction to some great mathematicians with their discoveries is given. In the last part of this paper relationship of mathematics and other disciplines is described.
\end{abstract}

\section{Mathematics in the words of some of pioneers of the field}

Galileo Galilei defined mathematics as, "The great book of nature can be read only by those who know the language in which it was written. And that language is mathematics." adding "Math is the way to understand all sorts of things in the world around us." According to Kant," Mathematics is a Science of all Sciences and art of all arts." In the words of Benjamin Peirce, mathematics can be defined as, "The science that draws necessary conclusions". Carl Friedrich Gauss said it, "The Queen of the Sciences". Davit Hilbert well said that, we are not speaking here of arbitrariness in any sense. Mathematics is not like a game whose tasks are determined by arbitrarily stipulated rules. Rather, it is a conceptual system possessing internal necessity that can only be so and by no means otherwise. Albert Einstein defined mathematics as, "As far as the laws of mathematics refer to reality, they are not certain; and as far as they are certain, they do not refer to reality." According to Claire Voisin, "There is creative drive in mathematics; it's all about movement trying to express itself."

\subsection{Aryabhata}

\section{Some Great Mathematicians}

It is no doubt that the world today is greatly indebted to the contributions made by Indian mathematicians. One of the most important contributions made by them was the introduction of decimal system as well as the invention of zero. Here are some the famous Indian mathematicians dating back from Indus Valley civilization and Vedas to Modern times. Aryabhata worked on the place value system using letters to signify numbers and stating qualities. He discovered the position of nine planets and stated that these planets revolve around the sun. He also stated the correct number of days in a year that is 365 .

\subsection{Brahamgupta}

The most significant contribution of Brahmgupta was the introduction of zero (0) to the mathematics which stood for "nothing". He also suggested that when zero is added to a number or subtracted from a number, the number remains unchanged and a number multiplied by zero becomes zero.

\subsection{Pythagoras of Samos}

Greek Mathematician Pythagoras is one of the first great mathematicians, living around 570 to 495 BC. $\mathrm{He}$ is commonly credited with the Pythagorean Theorem within trigonometry. This theorem is playing a large part in modern measurements and technological equipment, as well as being the base of a large portion of other areas and theorems in mathematics. He could be called the founding father of modern mathematics.

\subsection{Andrew Wiles}

Andrew Wiles is most well known for his proof of Fermat's Last Theorem. He did 'invent' large portions of new mathematics for his proof of the theorem. Besides, his dedication is often admired by most, as he quite literally shut himself away for 7 years to formulate a solution. When it was found that the solution contained an error, he returned to solitude for a further year before the solution was accepted

\subsection{Isaac Newton and Wilhelm Leibniz}

These two have placed together as they are both often given the honour of being the 'inventor' of modern infinitesimal calculus, and as such have both made monolithic contributions to the field. Leibniz is often given the credit for introducing modern standard notation, notably the integral sign. He made large contributions to the field of Topology. Whereas all round genius Isaac Newton has, because of the grand scientific epic 
Principia, generally become the primary man hailed by most to be the actual inventor of calculus. Both mathematicians made considerable vast contributions in their own manner.

\subsection{Leonardo Pisano Blgollo}

Blgollo, also known as Leonardo Fibonacci, is perhaps one of the middle ages greatest mathematicians. Living from 1170 to 1250, he is best known for introducing the infamous Fibonacci Series to the western world. Although known to Indian mathematicians since approximately 200 BC, it was, nonetheless, a truly insightful sequence, appearing in biological systems frequently. This Fibonacci also contributed greatly to the introduction of the Arabic numbering system. Today he is considered a great player in the development of modern mathematics.

\subsection{Alan Turing}

Computer Scientist and Cryptanalyst Alan Turing is regarded by many, to be one of the greatest minds of the 20th Century. He made significant discoveries and created ground breaking methods of code breaking that would eventually aid in cracking the German Enigma Encryptions. He is considered one of the first true computer scientists. Furthermore, he wrote a range of brilliant papers on the subject of computing that are still relevant today, notably on Artificial Intelligence, on which he developed the Turing test which is still used to evaluate a computers 'intelligence'. Remarkably, he began in 1948 working with D. G. Champernowne, an undergraduate acquaintance on a computer chess program for a machine not yet in existence. He would play the 'part' of the machine in testing such programs.

\subsection{Rene Descartes}

French Philosopher, Physicist and Mathematician Rene Descartes is best known for his 'Cogito Ergo Sum' philosophy. Despite this, the Frenchman, who lived 1596 to 1650, made ground breaking contributions to mathematics. Alongside Newton and Leibniz, Descartes helped provide the foundations of modern calculus (which Newton and Leibniz later built upon), which in itself had great bearing on the modern day field. He is well known for the development of Cartesian Geometry, known to most as the standard graph (Square grid lines, $\mathrm{x}$ and $\mathrm{y}$ axis, etc.) and its use of algebra to describe the various locations on such. Before this, most geometers used plain paper (or another material or surface) to preform their art. Previously, such distances had to be measured literally, or scaled. With the introduction of Cartesian Geometry this changed dramatically, points could now be expressed as points on a graph, and as such, graphs could be drawn to any scale, also these points did not necessarily have to be numbers. The final contribution to the field was his introduction of superscripts within algebra to express powers. And thus, like many others in this list, contributed to the development of modern mathematical notation.

\subsection{Euclid}

Living around 300BC, he is considered the Father of Geometry and his magnum opus: Elements, is one the greatest mathematical works in history, with its being in use in education up until the 20th century. Euclid is credited with the instruction of the rigorous, logical proof for theorems and conjectures. Such a framework is still used to this day, and thus, arguably, he has had the greatest influence of all mathematicians on this list. Alongside his Elements were five other surviving works, thought to have been written by him, all generally on the topic of Geometry or Number theory. There are also another five works that have, sadly, been lost throughout history.

\subsection{G. F. Bernhard Riemann}

Bernhard Riemann, born to a poor family in 1826, would rise to become one of the world's prominent mathematicians in the 19th Century. The list of contributions to geometry is large, and he has a wide range of theorems bearing his name. To name just a few: Riemannian geometry, Riemannian Surfaces and the Riemann Integral. However, he is perhaps most famous (or infamous) for his legendarily difficult Riemann Hypothesis; an extremely complex problem on the matter of the distributions of prime numbers.

\subsection{Carl Friedrich Gauss}

The 'Prince of Mathematics', made his first major discovery whilst still a teenager, and wrote the incredible Disquisitiones Arithmeticae, his magnum opus, by the time he was 21. Many know Gauss for his outstanding mental ability - quoted to have added the numbers 1 to 100 within seconds whilst attending primary school (with the aid of a clever trick). The local Duke, recognizing his talent, sent him to Collegium Carolinum before he left for Gottingen (at the time it was the most prestigious mathematical university in the world, with many of the best attending). After graduating in 1798 (at the age of 22), he began to make several important contributions in major areas of mathematics, most notably number theory (especially on Prime numbers). He 
went on to prove the fundamental theorem of algebra, and introduced the Gaussian gravitational constant in physics, as well as much more - all this before he was 24 !, he continued his work up until his death at the age of 77 , and had made major advances in the field which have echoed down through time.

\subsection{Leonhard Euler}

If Gauss is the Prince, Euler is the King. Living from 1707 to 1783 , he is regarded as the greatest mathematician to have ever walked this planet. It is said that all mathematical formulas are named after the next person after Euler to discover them. In his day he was ground breaking and on par with Einstein in genius. His primary contribution to the field is with the introduction of mathematical notation including the concept of a function (and how it is written as $\mathrm{f}(\mathrm{x})$ ), shorthand trigonometric functions, the 'e' for the base of the natural logarithm (The Euler Constant), the Greek letter Sigma for summation and the letter '/i' for imaginary units, as well as the symbol pi for the ratio of a circles circumference to its diameter. All of which play a huge bearing on modern mathematics, from the everyday to the incredibly complex.

As well as this, he also solved the Seven Bridges of Koenigsberg problem in graph theory, found the Euler Characteristic for connecting the number of vertices, edges and faces of an object, and (dis)proved many well known theories, too many to list. Furthermore, he continued to develop calculus, topology, number theory, analysis and graph theory as well as much, much more - and ultimately he paved the way for modern mathematics and all its revelations. It is probably no coincidence that industry and technological developments rapidly increased around this time.

\subsection{Srinivasa Ramanujan}

One of the celebrated Indian mathematicians.His important contributions to the field include Hardy Ramanujan-Littlewood circle method in number theory, Roger-Ramanujan's identities in partition of numbers, work on algebra of inequalities, elliptic functions, continued fractions, partial sums and products of hyper geometric series.

\section{Relationship between mathematics and other disciplines}

Mathematicians also work in many other fields, like: Astronomy and space exploration, Climate study, Medicine, National security, Robotics, Animated films and in a wide range of businesses

\subsection{Mathematics and Science}

According to Kant "A natural Science is a Science in so far as it is mathematical". And Mathematics has played a very important role in building up modern Civilization by perfecting all Science. There are uses of mathematics in all the "hard" sciences, such as biology, chemistry, and physics; the "soft" sciences, such as economics, psychology, and sociology; engineering fields, such as civil, mechanical, and industrial engineering; and technological fields such as computers, rockets, and communications. There are even uses in the arts, such as sculpture, drawing, and music. In addition, anything which uses a computer uses mathematics. Mathematics is about pattern and structure; it is about logical analysis, deduction, calculation within these patterns and structures. When patterns are found, often in widely different areas of science and technology, the mathematics of these patterns can be used to explain and control natural happenings and situations. Mathematics has a pervasive influence on our everyday lives, and contributes to the wealth of the country.

\subsection{Mathematics in Medicine}

Mathematics plays a huge role in medicine in many ways, in its clinic practice as well as in the scientific understanding of disease. In clinical practice, maths is important in quantifying subjective clinical details such as obesity and pain. Medical Professionals use maths while drawing statistical graphs of epidemics or success rates of treatment. Maths applies to X-rays and CAT-Scans. One another application involves a lithotripter. This is a medical device that uses property of an ellipse to treat gallstones and kidney stones.

\subsection{Rhythm of Mathematics in music}

There is an enormous and natural relation between the two. Music is all about seven notes ( $\mathrm{Sa}, \mathrm{Re}, \mathrm{Ga}$, $\mathrm{Ma}, \mathrm{Paa}, \mathrm{Dhaa}, \mathrm{Ni}, \mathrm{Saa})$. Mathematics describes the way how these notes should be sung or played on any instrument. Rhythm of Music comes from mathematics (Fibonacci numbers and golden ratio).

\section{Conclusion}

In this paper, various aspects of mathematics are discussed. Starting with definition of mathematics by some great mathematicians with the names of their discoveries in the field of mathematics; the relationship between mathematics and other disciplines is given. 


\section{References}

[1]. $\quad \underline{\text { www.wikipedia.com }}$

[2]. www.google.com

[3]. www.listverse.com

[4]. A Mathematician's Apology, by G. H. Hardy. Memoir, with foreword by C. P. Snow., Cambridge University Press, 1992.

[5]. H., Paul, I Want to Be a Mathematician. Springer-Verlag 1985

[6]. W.,Dunham, The Mathematical Universe. John Wiley 1994. 